\title{
Topographic Identification of High-Risk Valvular Regions for Patients With Calcific Degenerative Aortic Valve Stenosis
}

\author{
John A Sims ${ }^{1}$, Rodrigo Castaldoni ${ }^{1}$, Jose de Arimateia Araujo-Filho ${ }^{2}$, Roberto Victor ${ }^{2}$, Thamara \\ Morais $^{2}$, Antonio de Santis ${ }^{2}$, Pedro Lemos ${ }^{2}$, Flavio Tarassouti ${ }^{2}$, Marcelo Vieira ${ }^{2}$, João L Salinet ${ }^{1}$, \\ Antonildes N Assuncao-Jr ${ }^{2}$, Cesar Higa Nomura ${ }^{2}$ \\ ${ }^{1}$ Biomedical Engineering, Engineering Modelling and Applied Social Sciences Center, \\ UFABC, São Paulo, Brazil \\ ${ }^{2}$ Heart Institute (InCor), University of São Paulo Medical School, São Paulo, Brazil
}

\begin{abstract}
Features of calcific leaflet distribution from CT valve images may allow prediction of hemodynamic disease severity in calcific degenerative aortic valve stenosis (DAS). The proposed study describes a signal processing scheme for selecting valve areas from maximum intensity projection valve images in a cohort of $52+43$ patient images, diagnosed as having severe and moderate DAS. First the valve center and perimeter are approximated by a manually determined circle of radius $r$ and center $(x, y)$. The circle is then used to define eight masks, based on concentric circles of radius $\frac{r}{2}, \frac{r \sqrt{2}}{2}, \frac{r \sqrt{3}}{2}$ and $r$, each with center $(x, y)$. The masks are used to define pixel regions within the valvular area, and statistical and textural descriptors are applied to each. Sensitivity/ specificity testing is performed with these descriptors, applied to the pixels within each mask, which show that disease severity is best predicted by using the smallest, most central mask and statistical features of skewness and kurtosis, providing area under the curve of 0.844 and 0.840 respectively. Our methodology was simple to implement and use, and provided good discriminatory power for disease severity. It also overcomes some difficulties in an earlier method, since our solution is scalable to variation in aortic valve size and tests a range of statistical and textural descriptors.
\end{abstract}

\section{Introduction}

Aortic stenosis is the most common valvular heart disease in Europe and North America, with high prevalence $(2-7 \%)$ in the population aged above 65 years. Its most common cause in adults is calcification of a normal trileaflet [1], characterized pathologically by large nodular calcific masses within the aortic cusps [2].

De Santis et al. [3] investigate the hypothesis of whether the location of valve calcification influences the functional severity of DAS. Using a single maximum intensity projection (MIP) image for each patient, Figure 1, they segment the aortic valve and define a central circular region and a peripheral region. They calculate the mean attenuation in each region (HU), compute the Center/Periphery attenuation ratio, and show that this is a good predictor of moderate / severe aortic stenosis.

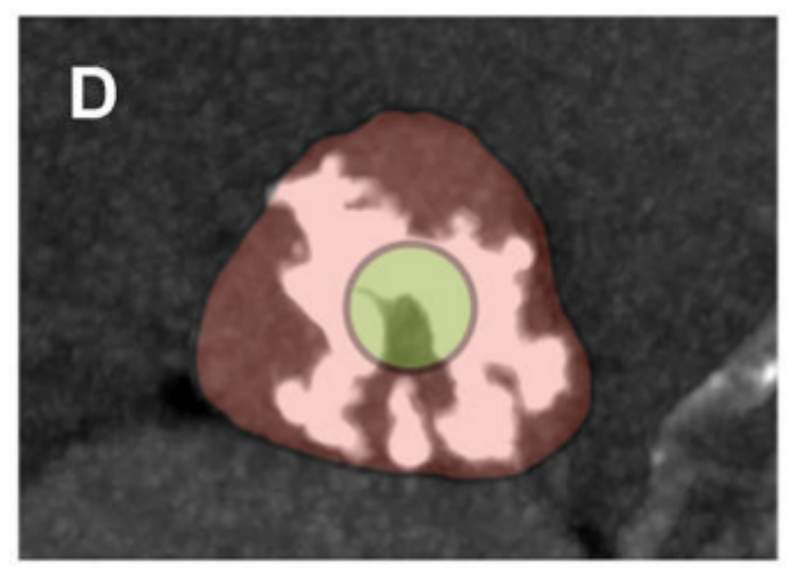

Figure 1: Low-contrast MDCT image. Aortic valve annulus plane was identified and a $10-\mathrm{mm}$ maximal intensity projection from the annulus plane towards the ascending aorta. The valve was manually segmented in the resulting short axis image (combined green and red region) and the resulting area divided into central circular regions of 1.0 $\mathrm{cm}^{2}$ (green) and a peripheral region (red) [3] figure

However, we note that (i) the manual segmentation of the aortic valve is time-consuming; (ii) a constant $1 \mathrm{~cm}^{2}$ central area is used across their analysis, whereas the area of the aortic valve varies between subjects, since valvular size is related to body surface; (iii) the statistical analysis is simple, while there exists a range of pixel-based features 

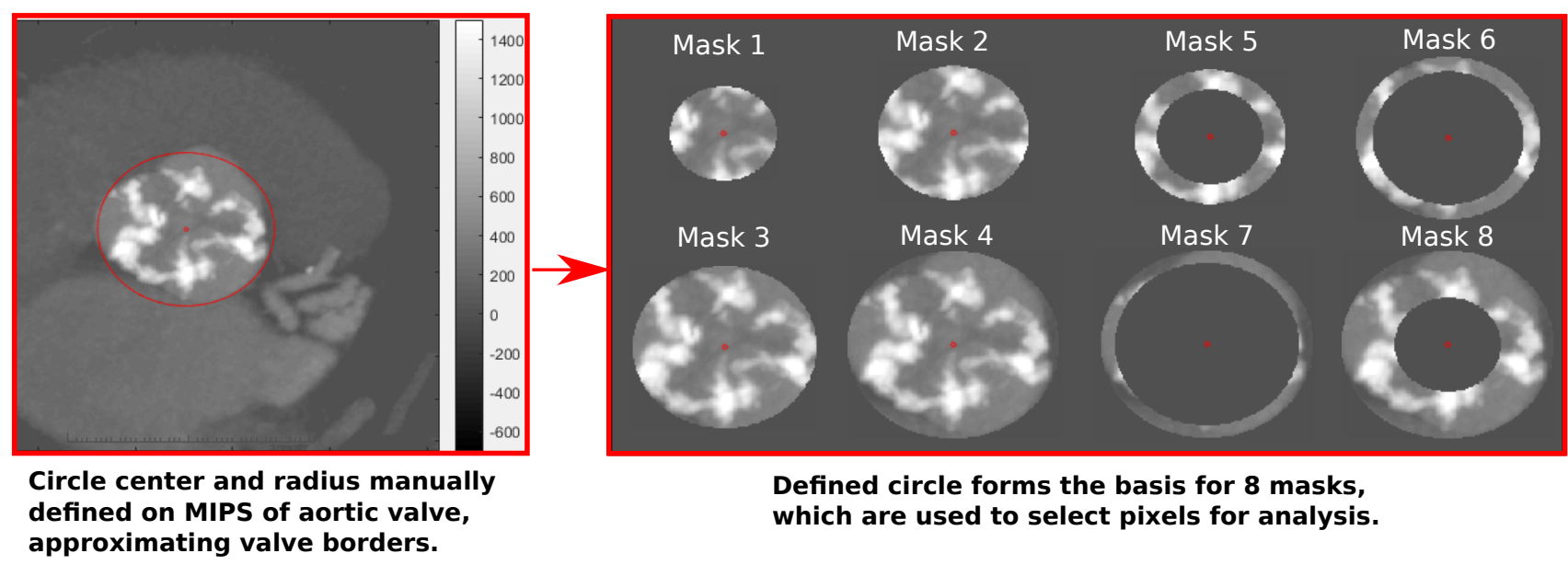

Defined circle forms the basis for 8 masks, which are used to select pixels for analysis.

Figure 2: Definition of mask areas from low-contrast MDCT image: (Left) Short-axis image from 10-mm MIP from the annulus plane towards the ascending aorta. (Right) Pixel regions after application of masks 1 to 8 (Table 1). figure

which could provide better classification of disease severity.

Our objective was to develop image processing software to improve disease classification, based on the above observations. First, accelerate the segmentation of the aortic valve region in the MIP image with a simple and feasible processing methodology. Second, develop more sophisticated analyses of regional distributions of calcific deposits. Third, create a methodology which is capable of automatically scaling the analysis region depending on the size of the aortic valve.

\subsection{Materials}

Patients with moderate or severe DAS were enrolled in the study and images acquired using the protocol described elsewhere [3]. (52+43) Patient images were acquired, respectively diagnosed as having severe and moderate DAS, using a 320-multidetector CT with low-dose contrast $(0.5 \mathrm{ml} / \mathrm{kg})$. Severe AS was diagnosed using transthoracic echocardiogram according to the presence of any of the following: aortic valve area $=1.0 \mathrm{~cm}^{2}$; mean gradient $=40 \mathrm{mmHg}$; peak aortic jet velocity $=4.0 \mathrm{~m} / \mathrm{s}$. Patients with moderate DAS did not have any criterium for severe AS and at least one of the following: aortic valve area 1.0$1.5 \mathrm{~cm}^{2}$; mean gradient $25-40 \mathrm{mmHg}$; peak aortic jet velocity $3.0-4.0 \mathrm{~m} / \mathrm{s}$.

A $10 \mathrm{~mm}$ maximum intensity projection (MIP) was created for the leaflet cross-section, including the entire leaflet depth.

Image processing routines were written using Matlab 9.2 (Natwick, Math Works Inc., 2017) and the entire processing took approximately 1 hour when run on a Windows 10 notebook with $16 \mathrm{~Gb}$ of RAM, i7 7th generation proces- sor, and a GeForce 940MX graphics card.

\section{Methodology}

Our image processing scheme was designed to approximate the aortic valve, divide it into different regions, and analyse the pixels contained in each.

(i) Valve segmentation was performed by manually approximating the valve circumference with a circle (of area, $A)$. First the circle center point is manually defined $(x, y)$ and then a second point is manually defined on the image to determine the radius, $r$ (Figure 2). The application of this circular mask to the image generates the image shown as Mask 4.

(ii) The division of pixels inside the circle into regions for analysis was performed by defining and applying eight masks. Masks 1 to 4 are circular regions, each centered on the manually defined center point but with different fractions of the circle with area $A$. The radii of circles in masks 1 to 4 , are given by $\frac{r}{2}, \frac{r \sqrt{2}}{2}, \frac{\sqrt{3}}{2} r, r$ respectively. Masks 5 to 7 are intersections of masks 1 to 4 , and mask 8 the periphery of mask 1 (Table 1 and Figure 2).

(iii) Analysis of the pixels in each region were performed using the Radiomics package [4] in R Studio version 1.1.463 [5,6]. First order statistical features (FOF), including mean, variance, skewness, kurtosis and those listed in Figure 4, were determined from pixel regions resulting from application of the 8 masks for all patients. Next, second order (textural) features were similarly extracted [7]. Area under the receiver operating characteristics (ROC) curve (AUC) [8] was used to evaluate the ability of each feature to predict DAS severity. 


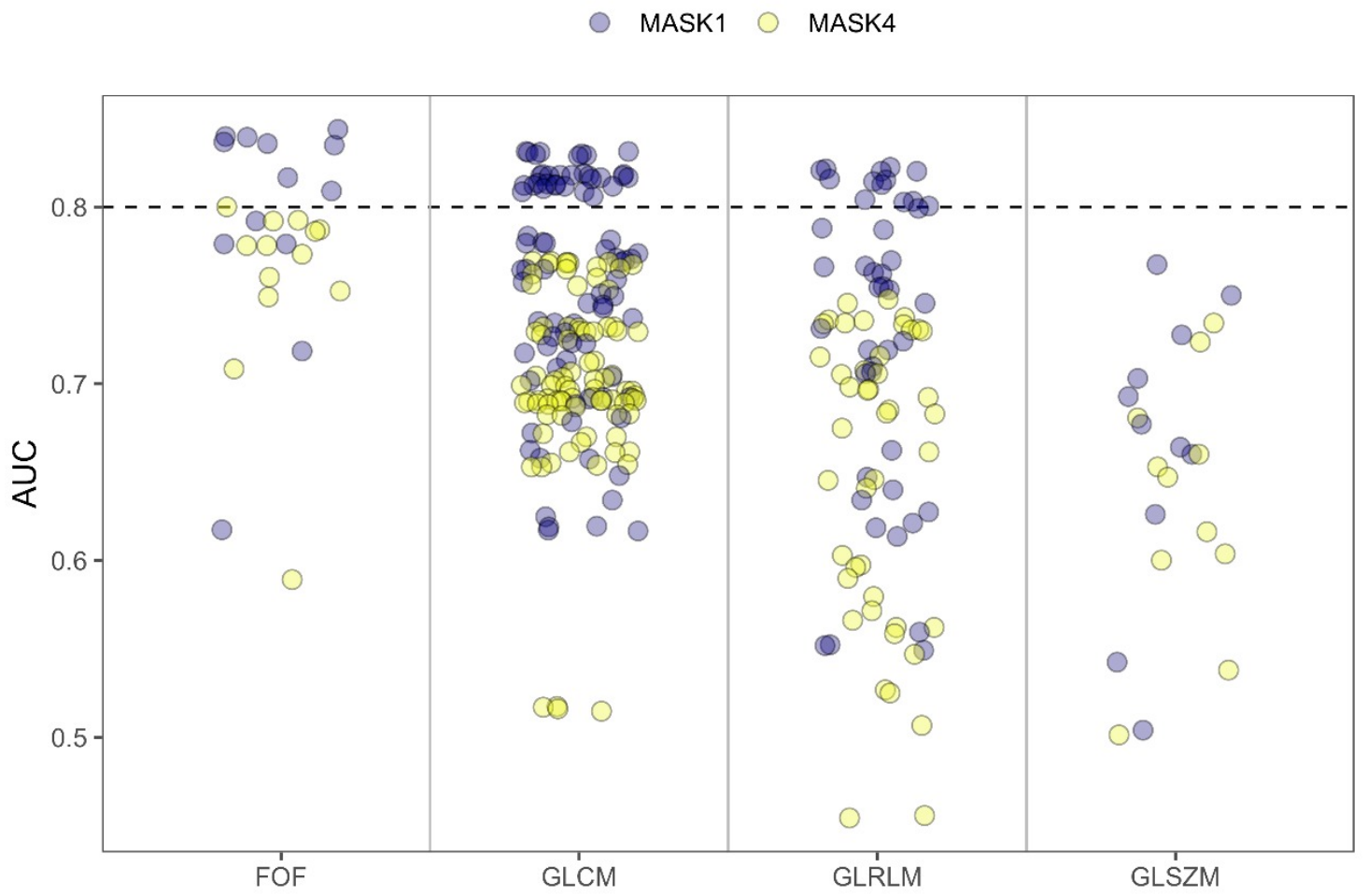

Figure 3: Performance (Area under the ROC Curve) for all features tested, separated by class (First Order Features (FOF), Gray Level Cooccurence Matrix (GLCM), Gray Level Run Length Matrix (GLRLM), Gray Level Size Zone Matrix (GLSZM)

figure

Table 1: Definition of mask areas. Masks 5 to 8 are described as the intersection of masks $2,3,4,4$ with the complement of 1,2, 3 and 1 respectively. table

\begin{tabular}{ccccc}
\hline Mask: & 1 & 2 & 3 & 4 \\
Area: & $\frac{1}{4} A$ & $\frac{1}{2} A$ & $\frac{3}{4} A$ & $A$ \\
& & & & \\
Mask: & 5 & 6 & 7 & 8 \\
Area: & $2 \bigcap 1^{C}$ & $3 \bigcap 2^{C}$ & $4 \bigcap 3^{C}$ & $4 \bigcap^{C}$ \\
\hline \hline
\end{tabular}

\subsection{Results}

All the features tested can be seen in Figure 3, where first-order features are seen to out-perform the textural features. Figure 4 provides a performance comparison between masks 1 and 4, and we see that the best features for predicting DAS were found in mask 1. Mask 1 was the best performer out of the eight masks tested.

The best performing features among those tested were Kurtosis $(\mathrm{AUC}=0.840)$ and skewness $(\mathrm{AUC}=0.844)$, ap- plied to mask 1.

\subsection{Discussion}

Our results show that statistical analysis of calcific leaflet distribution from $\mathrm{CT}$ valve images is able to predict hemodynamic disease severity in DAS, with the valve center seen as the most important region, an observation which is paralleled in the literature.

Our segmentation approach involved approximating the valve border with a circle, which is simpler and faster to execute than the manual segmentation of the border used in de Santis et al. We did not include independent selection of the central region in our software, but relied on a concentric circle approach. In certain images, where the center of the border circle does not match the meeting point of the leaflets, more sophisticated software or manual placement of the center might improve performance.

Our approach improves on the work by de Santis et al. by providing discriminatory performance and a methodology which is scalable to patients with aortic valves of various sizes. 


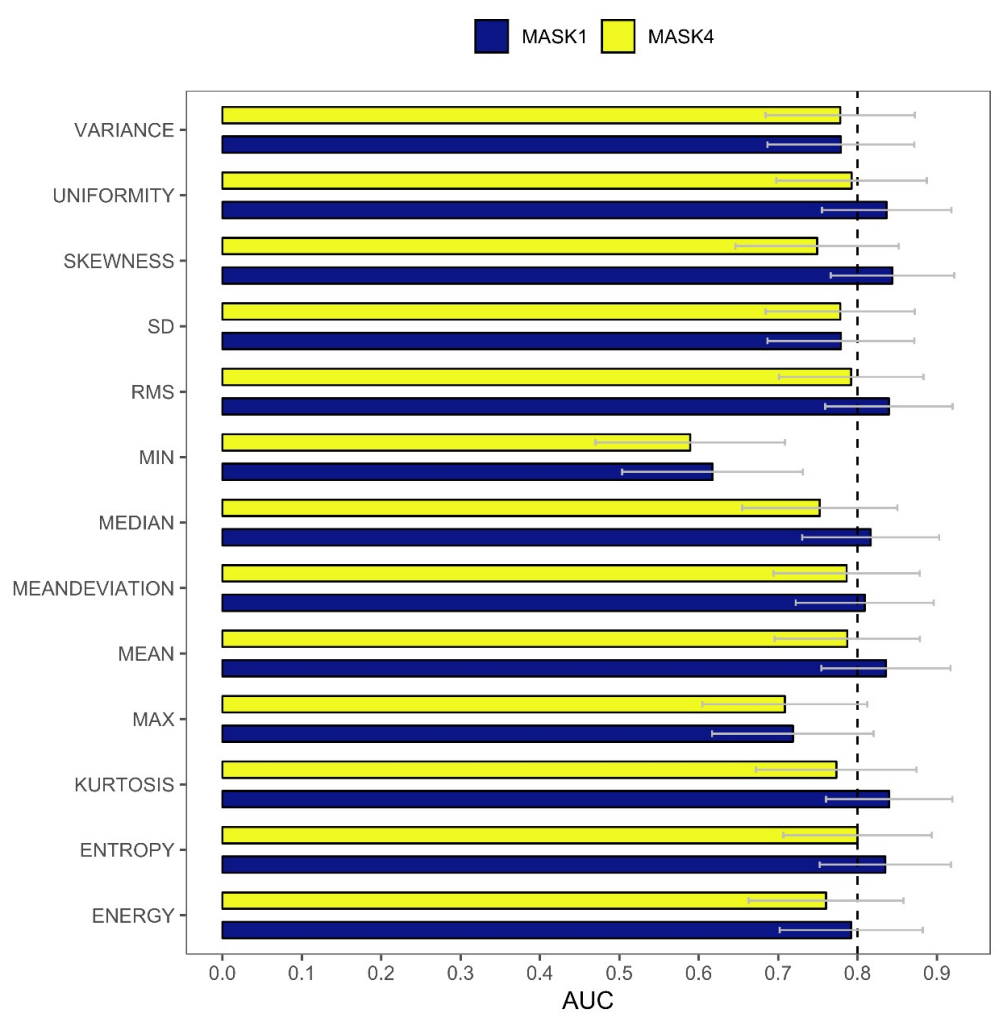

Figure 4: Best performing features after the AUC analysis, comparing mask 1 and 4 . Features extracted from mask 4 (entire valvular area) resulted, in every case, in lower performance than with mask 1 (central quarter area of valve). figure

Future work could investigate the predictive ability of masks with different areas, varying around 0.25 times the circle area.

\subsection{Conclusion}

The proposed method has been shown to be a simple, feasible and a powerful approach for identifying high-risk valvular regions for patients with calcific DAS.

\section{References}

[1] Ramaraj R, Sorrell VL. Degenerative Aortic Stenosis. BMJ 2008;336(7643):550-555.

[2] Rajamannan NM, Evans FJ, Aikawa E, Grande-Allen KJ, Demer LL, Heistad DD, Simmons CA, Masters KS, Mathieu P, O'Brien KD, Schoen FJ, Towler DA, Yoganathan AP, Otto CM. Calcific Aortic Valve Disease: Not Simply a Degenerative Process. Circulation 2011;124(16):1783-1791.

[3] de Santis A, Tarasoutchi F, Araujo Filho JdAB, Vieira MC, Nomura CH, Katz M, Spina GS, Sampaio RO, Accorsi TA, Rosa VE, Fernandes JR, Brown J, Edelman ER, Lemos PA. Topographic Pattern of Valve Calcification. JACC Cardiovascular Imaging 2017;23(7):829-838.
[4] Carlson J. Radiomics: Texture Analysis Matrices, $2016 . \quad$ URL https://github.com/joelcarlson/radiomics/. $\mathrm{R}$ package version 0.1.2.

[5] R Core Team. R: A Language and Environment for Statistical Computing. $\mathrm{R}$ Foundation for Statistical Computing, Vienna, Austria, 2015. URL https://www.R-project.org/.

[6] RStudio Team. RStudio: Integrated Development Environment for R. RStudio, Inc., Boston, MA, 2016. URL http://www.rstudio.com/.

[7] Haralick RM, Shanmugam K, Dinstein I. Textural Features for Image Classification. IEEE Transactions on Systems Man and Cybernetics 1973;SMC-3(6):610-621.

[8] Fawcett T. An Introduction to ROC Analysis. Pattern Recognition Letters 2006;27(8):861-874.

Address for correspondence:

Prof. Dr. John Andrew Sims Universidade Federal in ABC, UFABC

Alameda da Universidade s/n, Bairro Anchieta, São Bernardo do Campo, SP, CEP 09606-045, Brazil john.sims@ufabc.edu.br 